\title{
Desempenho cognitivo e equilíbrio funcional em idosos
}

\author{
Cognitive Performance and Functional Balance in elderly
}

\section{Giovanna Cristina dos Santos Caixeta ${ }^{1}$, Andreza Ferreira²}

\begin{abstract}
RESUMO
Objetivo. Comparar o desempenho cognitivo e o equilíbrio corporal funcional em idosos institucionalizados com os que vivem na comunidade. Método. A amostra consistiu de 25 idosos residentes na instituição de longa permanência de Patos de Minas - Vila Padre Alaor (G1) que não são submetidos a programa de atividade física e 25 idosos que vivem na comunidade e integrantes do projeto Unipam Sênior (G2) que inclui atividades físicas. O Mini Exame do Estado Mental - MEEM foi empregado para avaliar o desempenho cognitivo e a Escala de Equilíbrio de Berg para investigar o equilíbrio funcional. Para análise estatística dos dados utilizou-se o Teste de $t$ de Student para amostras independentes. Resultados. $O$ escore médio do teste do Mini Exame do Estado Mental entre os

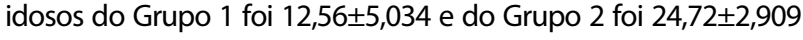
$(p<0,001)$. O escore médio da escala de Equilíbrio de Berg entre os idosos do Grupo 1 foi 44,92 $\pm 6,531$ ) e do Grupo 2 foi 52,40 $\pm 2,273$ $(p<0,001)$. Conclusão. Idosos institucionalizados que vivem em instituições que não oferecem programas de atividades físicas apresentam menor desempenho cognitivo e comprometimento do equilíbrio funcional em relação aos idosos que vivem na comunidade e praticam atividade física.
\end{abstract}

Unitermos. Equilíbrio Musculosquelético, Cognição, Idoso. Citação. Caixeta GCS, Ferreira A. Desempenho cognitivo e equilíbrio funcional em idosos

\section{SUMMARY}

Objective. To compare cognitive performance and functional balance in institutionalized elderly and those who live in the community. Method. The sample consisted of 25 elderly people leaving in an institution at Patos de Minas - Vila Padre Alaor (G1), and that are not enrolled in a program of physical activity, and 25 elderly people living in a community enrolled in the Unipam Senior project, including physical activity (G2). The Mini Mental State Examination (MMSE) was used to evaluate the cognitive performance and the Berg's Balance Scale was used to investigate the functional balance. The statistical analysis was carried out using the Student t-test for independent samples. Results. The MMSE mean score was $12.56 \pm 5.034$ to $G 1$ and $24.72 \pm 2.909$ to $G 2(p<0.001)$. The functional balance performance mean score was $44.92 \pm 6.351$ to $\mathrm{G} 1$ and $52.4 \pm 2.273$ to $\mathrm{G} 2$ ( $p<0.001)$. Conclusion. Institutionalized elderly people present lower cognitive performance and functional balance disorders than the elderly living in the community and practicing physical activity.

Keywords. Musculoskeletal Equilibrium, Cognition, Elderly. Citation. Caixeta GCS, Ferreira A. Cognitive Performance and Functional Balance in elderly.
Trabalho realizado no Centro Universitário de Patos de Minas UNIPAM, Patos de Minas-MG, Brasil.

1. Fisioterapeuta, Mestranda do Programa em Reabilitação Vestibular e Inclusão Social da Universidade Bandeirante de São Paulo - UNIBAN, Professora do Centro Universitário de Patos de Minas - UNIPAM, Patos de Minas-MG, Brasil.

2. Graduanda do curso de Fisioterapia do Centro Universitário de Patos de Minas - UNIPAM, Patos de Minas-MG, Brasil
Endereço para correspondência: R. Anicésio V Valadão, 30.

CEP 38702-064. Patos de Minas-MG E-mail: giovannacaixeta13@hotmail.com
Recebido em: 08/12/2008 Revisado em: 09/12/2008 a 10/08/2009

Aceito em: 11/08/2009

Conflito de interesses: não 


\section{INTRODUÇÃO}

$\mathrm{O}$ aumento das percentagens de idosos na população mundial mudou de maneira contundente as estratégias de abordagem de suas doenças. $O$ envelhecimento populacional é um fato mundial'. O indivíduo idoso, nos dias atuais consegue facilmente chegar à idade de 80 a 90 anos, entretanto, não consegue evitar os efeitos da senescência natural do qual lhe é atribuído. $O$ enveIhecimento é aquele período da vida que, sucede a maturidade e é caracterizado por declínio das funções orgânicas, e, em decorrência, acarreta maior susceptibilidade á eclosão de doenças ${ }^{2,3}$.

Na literatura, diversos estudos apontam que o envelhecimento gera alterações funcionais de postura e de equilíbrio corporal, processo este que pode ser acompanhado pelo declínio das capacidades físicas e cognitivas ${ }^{4}$. A presença dessas alterações no envelhecimento fisiológico pode ser um preditor para ocorrência de declínio cognitivo e demência que decorrem da degeneração neuronal e do decréscimo de plasticidade responsáveis pela menor efetividade do processamento central e da integração das informações sensoriais (visual, vestibular e somatossensorial), interferindo no controle postural ${ }^{5}$. O envelhecimento compromete a habilidade do sistema nervoso central em realizar o processamento dos sinais vestibulares, visuais e proprioceptivos responsáveis pela manutenção do equilíbrio corporal, bem como diminui a capacidade de modificações dos reflexos adaptativos. Com esses processos, ocorrem as vertigens e tonturas. Para maior parte de idosos independentes, os déficits de equilíbrio corporal não levam a diagnósticos específicos e sim a processos degenerativos mal definidos ${ }^{6}$.

O idoso pode apresentar alterações normais como lentificação de atividades motoras grossas, redução da habilidade em atividades motoras finas, declínio no desempenho de tarefas psicométricas e de atenção, principalmente relacionadas a tarefas motoras, com o aumento de tempo de reação. $O$ declínio desencadeado pelo envelhecimento incide especialmente nas tarefas que exigem rapidez, atenção, concentração e raciocínio indutivo ${ }^{5,6}$. As alterações e os déficits causados pelo declínio cognitivo levam ao declínio funcional, com diminuição e/ou perdas das habilidades para o desenvolvimento das atividades cotidianas, interferindo de forma significativa na realização das atividades de vida diária. Com a progressão do caso pode ocorrer dificuldade no seu autocuidado, comprometimento da linguagem, incapacidade de orientar-se e reconhecer fisionomias, alterações no sono e déficits de locomoção ${ }^{5,7,8}$.

Essas manifestações de desequilíbrios no idoso causam grande impacto, levando à redução de sua autonomia social, uma vez que acabam reduzindo suas atividades de vida diária, pela predisposição a quedas e fraturas, trazendo sofrimento, imobilidade corporal, medo de cair e altos custos com o tratamento de saúde ${ }^{8,9}$.

Com todas as alterações observadas nos idosos, estudiosos comprovaram que a melhor forma de prevenção de doenças está em uma atividade física adequada e regular. Ela garante a força muscular e a mobilidade das articulações que além de prevenir quedas, dá mais disposição, e favorece a auto-estima. Os maiores benefícios da atividade física são o aumento da flexibilidade, diminuição da gordura corporal, melhora do sono, controle da ansiedade, melhora da circulação e da capacidade cardiopulmonar, redução de perda óssea e melhora das dores articulares relacionadas à má postu$\mathrm{ra}^{10} \mathrm{e}$ podem também ajudar os idosos a permanecerem cognitivamente saudáveis.

Várias evidências sugerem que pessoas que se exercitam regularmente possuem menos chance de apresentar declínio cognitivo comparado pessoas do qual não se engajam em programas de exercício ou que não se exercitam regularmente. Estes estudos revelam que as atividades físicas podem ser eficazes em prevenir, retardar ou reverter o processo de fragilidade, que seria a instabilidade e o risco de perda de função funcional, mental, e física, observada em pessoas idosas ${ }^{11-13}$.

A prática de atividade física melhora o funcionamento cognitivo dos idosos ${ }^{14}$. Outro estudo mostra que a incidência de demência em idosos por um período médio de 6,2 anos foi na proporção de 13 para cada 1000 pessoas por ano entre os participantes que se exercitaram três ou mais vezes por semana, comparado com 19,7 para cada 1000 pessoas por ano entre os que se exercitaram menos de três vezes por semana ${ }^{15}$. Os autores do estudo sugeriram que a prática regular de atividade física está associada com redução do risco de desenvolver demência.

Poucos estudos avaliaram a relação entre atividade física e qualidade de vida em idosos ${ }^{16}$. Alguns demonstraram a melhora da qualidade de vida com a prática de exercício em ambiente de 
grupo, possivelmente promovido pelos efeitos de uma maior interação social ou melhora da capacidade funcional. Há necessidade de mais estudos empíricos que apóiem a relação entre prática de atividades físicas e melhor funcionamento cognitivo ${ }^{17}$. O mesmo pode-se dizer da necessidade de explicar a relação entre os efeitos benéficos do exercício físico e os transtornos mentais. Esta evidencia está apontada em diversos estudos que abordam os benefícios psicológicos da prática regular de atividade física. Porém, os estudos que tentam investigar os efeitos e mecanismos pelo qual o exercício físico pode promover melhoras psicológicas e fisiológicas nos transtornos mentais ainda são bastante reduzidos ${ }^{18,19}$.

Entretanto nem toda parcela da população idosa, como é o caso da maioria daqueles que são institucionalizados pode se beneficiar com as recomendações de como alcançar um envelhecimento saudável, resultante da interação entre saúde física, saúde mental, independência na vida diária, integração social, suporte familiar e independência econômica. O descaso mostrado pela sociedade, frente à maioria das instituições de longa permanência é evidenciado pela marginalização de muitos idosos que ali vivem. Ao serem deixados de lado, passam o restante de suas vidas como sujeitos passivos, sem utilidade para a sociedade, apenas aguardando um fim para toda a impotência e dependência em que vivem ${ }^{20}$.

Apesar da existência de leis e medidas práticas direcionadas ao idoso, como a Política Nacional do Idoso de 1994, a Política Nacional de Saúde do Idoso aprovada em 1999 e o Estatuto do Idoso de 2003, a implementação de políticas públicas em relação ao idoso institucionalizado em alguns casos isso não acontece. Quando se observam as diretrizes presentes nas políticas, percebe-se que os cuidados frente ao idoso continuam recaindo quase que exclusivamente sobre a família. Entretanto, apesar de priorizar o cuidado familiar e colocar a institucionalização em segundo plano, o Estado deve dar conta de buscar formas de promover, proteger e recuperar, igualmente o idoso institucionalizado ${ }^{19,21}$. Com base na Portaria SAS 73/01, as ILPI devem oferecer cuidados ao idoso, conforme o seu grau de dependência, criando serviços especializados.

No entanto, apesar das recomendações explícitas também na Política Nacional do Idoso, a realidade das instituições asilares brasileiras ainda apresenta estruturas deficitárias no que diz respeito aos cuidados com os idosos ${ }^{22}$. Inclusive pode-se perceber que grande parte das ILPI carece de condições propícias ao bem-estar de seus idosos, bem como de profissionais interessados em preservar e estimular os idosos a se manterem cognitivamente e fisicamente saudáveis.

Os asilos, geralmente, são casas inapropriadas e inadequadas às necessidades do idoso, tanto pela falta de recursos, pois muitas vezes esses asilos vivem apenas de doações, quanto pela grande quantidade de moradores, que às vezes ultrapassa a quantidade permitida. Ademais, esses locais vêm também dificultar as relações interpessoais no contexto comunitário, indispensáveis à manutenção do idoso pela vida e pela construção de sua cidadania e também constituí o convívio do idoso longe da família, favorecendo seu isolamento e sua inatividade física e mental. Os asilos apresentam espaços e áreas físicas semelhantes a grandes alojamentos, sem conforto, a maioria não possui pessoal especializado para assistência social e à saúde ou que possuam uma proposta de trabalho voltada para manter o idoso independente e autônomo ${ }^{23}$.

Sabendo do grande crescimento da população idosa nas últimas décadas, ressalta-se a necessidade da realização de pesquisas na área cognitiva e do equilíbrio corporal, que abordem as alterações concomitantes do equilíbrio e cognição ${ }^{24}$. Os estudos e pesquisas que fundamentam as relações entre o processamento cognitivo e as alterações do equilíbrio corporal são escassos na literatura. A natureza desta relação não foi completamente evidenciada. Isto ocorre talvez não somente pelo fato dos métodos de avaliação cognitiva serem diferenciados, como também pela dúvida sobre qual a natureza da relação entre o equilíbrio corporal e do processamento cognitivo de diferentes realidades dos idosos ${ }^{25}$. O objetivo o estudo é comparar o desempenho cognitivo e o equilíbrio corporal funcional em idosos institucionalizados e os que vivem na comunidade.

\section{MÉTODO}

O presente estudo é do tipo transversal único, onde foi avaliado e comparado o desempenho do equilíbrio funcional e aspectos cognitivos em 25 idosos com mais de 60 anos que residiam no Asilo Vila Padre Alaor (Grupo 1) e 25 idosos da comunidade frequentadores do pro- 
jeto UNIPAM Sênior do Centro Universitário de Patos de Minas - UNIPAM (Grupo 2). O estudo foi realizado nos meses de agosto a setembro de 2008 , sendo este aprovado pelo comitê de ética em pesquisa do Centro Universitário de Patos de Minas (5508). Foi submetido às coordenações de ambos os locais da pesquisa, uma carta convite de aceite e de responsabilidade sobre os idosos.

Para o cálculo do tamanho da amostral utilizou a análise on-line disponível no site http://lee. dante.br/pesquisa/amostragem/calculo_amostra. html considerado as seguintes estimativas obtidas com base bibliográficas. Desvio Padrão $=3$; Diferença a ser detectada: 3,0; Nível de significância: 5\%; Poder do teste: $90 \%$; Teste de hipótese: bicaudal;Tamanho da amostra calculado para cada grupo: 21 .

Para a composição da amostra foi adotado o sorteio aleatório dentro das populaçãoes selecionadas ao estudo e obedecendo aos critérios de inclusão: assinarem com o Termo de consentimento livre, pacientes com marcha independente, idosos com mais de 60 anos, ambos os gêneros; e critérios de exclusão: não concordarem com o Termo de consentimento livre, tomarem medicamentos que alteram o sistema nervoso central, serem portadores de doenças degenerativas osteomusculares com quadro avançado, fazer uso de muletas, bengalas ou andadores, possuírem limitações cognitivas graves e apresentem comprometimento visual e/ou auditivo grave.

Os integrantes da pesquisa foram avaliados pela estagiária do último ano do curso de fisioterapia e pela orientadora técnica científica do projeto. Em um primeiro momento, os pacientes passaram por uma anamnese breve, com os dados clínicos gerais do idoso pertencente a cada grupo, como idade, gênero, bem como a pratica ou não de atividade física e nível de escolaridade de cada grupo. Para o Grupo 1, visando não desgastar os idosos a primeira parte do formulário, foi respondida pelas enfermeiras responsáveis das instituições, devido às características das questões. Para o Grupo 2, as questões gerais da anamnese foram respondidas pelos próprios idosos.

A próxima etapa da avaliação consistiu em avaliar a capacidade cognitiva de ambos os grupos onde foi adotado o Mini Exame do Estado Mental, que envolve questões como orientação espacial, memorização, atenção, cálculo e linguagem, com pontuações que alcança um máximo de 30 pontos $^{26}$. Foi escolhido este teste por ser um teste sim- ples e ao mesmo tempo fornecer imediatamente um indicador razoavelmente indicador de demência. É considerado ter alta especificidade sem perder muito a sensibilidade, quando se utiliza o ponto de corte padrão 23 pontos para pessoas com escolaridade acima de 9 anos, indicando um padrão de normalidade, e abaixo deste valor indicando algum tipo de demência, necessitando assim uma investigação mais minuciosa, enquanto o corte de 17 pontos foi ótimo para aqueles com menos escolaridade, sendo abaixo deste valor demonstrando a presença de alterações significativas indicando a necessidade de aplicações de outros testes ${ }^{24-27}$.

Posteriormente foram avaliados segundo a escala de equilíbrio de Berg $^{28}$, constituída por uma escala de 14 tarefas comuns que envolvem o equilíbrio estático e dinâmico tais como alcançar, girar, transferir-se, permanecer em pé e levantarse. A realização das tarefas é avaliada por meio de observação e a pontuação varia de $0-4$, totalizando um máximo de 56 pontos. Cada tarefa funcional é graduada em uma escala ordinal de 0 (não consegue realizar) a 4 (desempenho normal), atingindo um escore total de 56 pontos. Como ponto de corte, utilizamos o escore de 45 pontos, cujos valores menores são indicativos do uso de dispositivos de auxílio à marcha ${ }^{28}$. É necessário demonstrar cada tarefa e/ou instruir o sujeito da maneira em que está escrito abaixo. Quando reportar a pontuação, registre a categoria da resposta de menor pontuação relacionada a cada item. Os equipamentos necessários são um cronômetro (ou relógio comum com ponteiro dos segundos) e uma régua ou outro medidor de distância com fundos de escala de $5 \mathrm{~cm}, 12,5 \mathrm{~cm}$ e $25 \mathrm{~cm}$ e uma cadeira de braços. Na maioria dos itens, pede-se ao sujeito manter uma dada posição por um tempo determinado. Progressivamente, mais pontos são subtraídos caso o tempo ou à distância não sejam atingidos, caso o sujeito necessite de supervisão para a execução da tarefa, ou se o sujeito apóiase num suporte externo ou ainda se ele recebe ajuda do examinador. É importante que se torne claro aos sujeitos que estes devem manter seus equilíbrios enquanto tentam executar a tarefa. A escolha de qual perna permanecerá como apoio e o alcance dos movimentos fica a cargo dos sujeitos. Julgamentos inadequados irão influenciar negativamente no desempenho e na pontuação.

Para análise estatística dos dados, foi utilizado o teste de $\mathrm{t}$ de Student para amostras 
independentes para comparar o nível de equilíbrio e cognição, entre o Grupo 1 e o Grupo 2. Foi utilizado o teste Exato de Fisher para a comparação a escolaridade e os grupos. Foi utilizado o programa SSPS, versão 12. Através do teste de normalidade Shapiro-Wilk, foi confirmada a existência de normalidade para as variáveis estudadas entre os grupos testados $(p>0,05)$.

\section{RESULTADOS}

A amostra deste estudo foi constituída por 50 idosos, onde 25 pertenciam ao Grupo 1 (institucionalizados) e 25 ao Grupo 2 (idosos da comunidade). A idade média dos indivíduos foi de $72,48 \pm 8,05$
A maioria dos idosos (48\%) do Projeto Unipam Sênior tinham escolaridade primária e a maioria do grupo da Vila Padre Alaor (80\%) não tinham escolaridade ( $p<0,001$, Tabela 1). O escore médio do teste do Mini Exame do Estado Mental entre os idosos do Grupo 1 foi 12,56 $\pm 5,034$ e do Grupo 2 foi $24,72 \pm 2,909$ ( $p<0,001$, Tabela 2). O escore médio da escala de Equilíbrio de Berg entre os idosos do Grupo 1 foi $44,92 \pm 6,531$ ) e do Grupo 2 foi $52,40 \pm 2,273$ ( $p<0,001$; Tabela 3).

Tabela 1- Comparação entre a Escolaridade entre os idosos do projeto Unipam Sênior e idosos da Vila Padre Alaor.

\begin{tabular}{|c|c|c|c|c|c|c|}
\hline \multirow{2}{*}{$\begin{array}{l}\text { Grupo } \\
\text { Experimental }\end{array}$} & \multicolumn{6}{|l|}{ Escolaridade } \\
\hline & Primáric & Fundament & Médic & Superiol & Nenhum & \\
\hline $\begin{array}{l}\text { Projeto Unipam } \\
\text { Sênior }\end{array}$ & $12(48 \%)$ & $6(24 \%)$ & $5(20 \%$ & $2(8 \%)$ & -- & $25(100 \%$ \\
\hline Vila Padre Alaor & $5(20 \%)$ & -- & -- & - & $20(80 \%)$ & $25(100 \%$ \\
\hline Total & $17(34 \%)$ & $6(12 \%)$ & $5(10 \%$ & $2(4 \%)$ & $20(40 \%)$ & $50(100 \%$ \\
\hline
\end{tabular}

Teste Exato de Fisher: $p<0,001$

Tabela 3 - Comparação entre os escores médios da Escala de Equilíbrio de Berg entre os idosos do projeto Unipam Sênior e idosos da Vila Padre Alaor.

\begin{tabular}{lllll}
\hline Grupo Experimental & Média & Desvio Padrão & T calculado & Significância \\
\hline Vila Padre Alaor & 44,92 & 6,531 & & \\
& & & & \\
Projeto Unipam Sênior & 52,40 & 2,273 & 5,408 & $<\mathbf{0 , 0 0 1}$ \\
\hline
\end{tabular}

Tabela 2 - Comparação entre os escores médios do Mini Exame do Estado Mental entre os idosos Vila Padre Alaor (Grupo 1) e Projeto Unipam Sênior (Grupo 2 ):

\begin{tabular}{lllll}
\hline Grupo Experimental & Média & Desvio Padrão & T calculado & Significância \\
\hline Vila Padre Alaor & 12,56 & 5,034 & & \\
Projeto Unipam Sênior & 24,72 & 2,909 & 10,458 & $<0,001$
\end{tabular}




\section{DISCUSSÃO}

O estudo objetivou avaliar o desempenho do equilibrio corporal e da cognição entre idosos com diferentes estilos de vida. Em relação ao nível de cognição, os resultados mostraram uma diferença significativa entre os idosos que vivem na sociedade e praticam algum tipo de atividade e os idosos que vivem reclusos e longe das famílias e não praticam atividades físicas. 0 grupo1, mostrou um nível de escolaridade mais baixo do que o Grupo 2, que reflete nos baixos escores obtidos no teste do MEEM, com média de 12,56, sendo que autores ${ }^{24-27}$ indicam como nota de corte para idosos com baixa escolaridade a nota de 17 pontos. Isto demonstra claramente que a institucionalização leva à perda da capacidade cognitiva e ainda a falta de incentivos a prática de atividades intelectuais também prejudica o desenvolvimento da memória e raciocínio. Os escores mais baixos obtidos pelo grupo de institucionalizado podem ser explicados pela falta de escolaridade e pela própria institucionalização, que acaba por contribuir de modo importante para a degradação cognitiva ${ }^{25}$. A prática de atividade física meIhora o funcionamento cognitivo dos idosos ${ }^{14}$. Os idosos do Grupo 1 não possuem incentivos para a prática regular de atividade física, controlada por profissionais de saúde apesar da existência de leis e medidas práticas direcionadas ao idoso, como a Política Nacional do Idoso de 1994, a Política Nacional de Saúde do Idoso aprovada em 1999 e o Estatuto do Idoso de 2003. No caso específico da cidade de Patos de Minas, observouse que a implementação de políticas públicas em relação ao idoso institucionalizado ainda não recebeu o tratamento adequado pelos poderes públicos, o que se evidencia quando se compara os escores obtidos pelo Mini Exame do Estado Mental (MEEM) e pela avaliação do equilíbrio corporal, pela escala de BERG, do grupo1 em relação ao grupo 2.

Idosos institucionalizados, em geral, dispõem de poucas atividades físicas para participarem, o que pode agravar o ciclo vicioso: enveIhecimento, sedentarismo e déficit da capacidade funcional ${ }^{29}$. Para os idosos integrantes do projeto Unipam Sênior, que oferece atividades das mais diversas áreas, como aulas de dança, hidroginástica, artesanato e computação, observa-se o bom desempenho dos idosos, bem como estão sempre estimulados ao desenvolvimento de atividades intelectuais e físicas. A freqüência dos idosos a essas atividades é controlada por uma equipe de educadores físicos. Os idosos do grupo 2 são acompanhados e constantemente avaliados por uma equipe multidisciplinar de profissionais, em que se inclui médico clínico, psicólogo e fisioterapeuta.

Em relação ao equilíbrio corporal, constatou-se também uma diferença significativa entre os dois grupos. Além disso, os melhores escores foram encontrados no Grupo 2, onde os idosos praticantes de atividade física, não só melhoram o equilíbrio, mas também os torna mais sociáveis $^{14}$. O Grupo 1, além de não se exercitarem, também não possui incentivo e participações ativas em incentivo a pratica de atividade física por profissionais da área de saúde. Para os idosos institucionalizados, haverá benefícios quando estes forem submetidos à prática da abordagem motora regular e sistematizada, bem como medidas de prevenção, proteção bem como de estimulação motora e cognitiva ${ }^{30}$. Isto fica evidente desde que sabemos que a atividade física adequada e regular é a melhor forma de prevenção de doenças, garantindo a força muscular e a mobilidade das articulações, prevenindo as quedas, favorecendo maior disposição, e estimulando a auto-estima. Além disso, os achados deste estudo contribuem para estimular outros estudos que visem a analisar os mecanismos dos aspectos cognitivos e do equilíbrio corporal nos idosos e suas inter-relações de como isto se processa com os benefícios da atividade física. Com base em todos os resultados fica clara a importância da implantação de um programa de reabilitação na vida de idosos.

\section{CONCLUSÃO}

A pesquisa mostrou que os idosos institucionalizados em instituições que não planejam programas de atividades físicas apresentam menor desempenho cognitivo e comprometimento do equilíbrio funcional em relação aos idosos que vivem na comunidade e praticam atividade física. Isto mostra que a implementação de programas de promoção a saúde nas instituições de longa permanência podem trazer benefícios aos idosos institucionalizados. 


\section{REFERÊNCIAS}

1.Kalache A, Veras RP, Ramos LR. O envelhecimento da população mundial: um desafio novo. Rev Saúde Pub1987;21:200-0. 2.Netto MP, Brito FC. Urgências em geriatria: Epidemiologia Fisiopatologia- Quadro clínico- controle terapêutico. São Paulo: Atheneu, 2001, 476p.

3.Simoceli L, Bittar RMS, Bottino MA, Bento RF. Perfil diagnóstico do idoso portador de desequilíbrio corporal: resultados preliminares. Rev Bras Otorrinolaringol 2003;69:772-7.

4.Pereira SRM. O idoso que cai. In: Guedes SL. Sociedade Brasileira de Geriatria e Gerontologia. Caminhos do Envelhecer. Rio de Janeiro: Revinter, 1994, p217-23.

5.Argimon IIL, Stein LM. Habilidades cognitivas em indivíduos muito idosos: um estudo longitudinal. Rev Saúde Pub 2005;21:64-72.

6.Kauffman TL. Manual de Reabilitação Geriátrica. Rio de Janeiro: Guanabara Koogan, 2001, 389p.

7.Kato EM, Radanovic M. Fisioterapia nas demências. Rio de Janeiro: Atheneu, 2007, 232 p.

8.Ruewer SL, Rossi AG, Simon LF. Equilíbrio no idoso. Rev Bras Otorrinolaringol 2005;71:298-303.

9.Oliveira SFD, Duarte YAO, Lebrão ML, Laurenti R. Demanda referida e auxílio recebido por idosos com declínio cognitivo no município de São Paulo. Rev Saúde Socied 2007;16:81-9.

10.Caldas CP. Cuidado Familiar. In: Veras R, Lourenço R. Formação Humana em Geriatria e Gerontologia: Uma Perspectiva interdisciplinar. Rio de Janeiro: UNATI/UERJ, 2006, p 335-9. 11 Butler RN, Forette F, Greengross BS. Maintaining cognitive health in an ageing society. J Royal Soc Prom Health 2004; 124:119-20.

12.Ferrucci L, Guralnik JM, Studenski S, Fried LP, Cutler GB Jr, Walston JD. Designing randomized, controlled trials aimed at preventing or delaying functional decline and disability in frail, older persons: a consensus report. J Am Geri 2004;52:625-34. 13.Faber MJ, Bosscher RJ, Paw ACJM, Wieringen PCV. Effects of Exercise Programs on Falls and Mobility in Frail and Pre-Frail Older Adults: A Multicenter Randomized Controlled Trial. Arc Phys Med Rehab 2006;87:885-96.

14.Shephard RJ. Envelhecimento, Atividade Física e Saúde. São Paulo: Phorte, 2003, 496 p.

15.Larson EB, Wang L, Bowen JD, McCormick WC, Teri L, Crane $P$, et al. Exercise Is Associated with Reduced Risk for Incident Dementia among Persons 65 Years of Age and Older. An Inter Med 2006;144:73-81.

16.Drewnowski A, Monsen E, Birkett D, Gunther S, Vendeland $\mathrm{S}$, Su J, et al. Heatlh Screening and Health Promotion Programs for the Elderly. Dis Man Health Outc 2003;11:299-309.
17.Tomporowski PD. Effects of acute bouts of exercise on cognition. Acta Psychol 2003; 112:297-324.

18.Araújo SRC, Mello MT, Leite JR. Anxiety disorders and physical exercise. Rev Bras Psiquiatr 2007;29:164-71.

19.Antunes HKM, Santos RF, Casilhas R, Santos RVT, Bueno OAF, Mello MT. Exercício físico e função cognitiva: uma revisão. Rev Bras Med Esp 2006;12:108-14.

20.Ramos LR. Fatores determinantes do envelhecimento saudável em idosos residentes em centro urbano: Projeto Epidoso, São Paulo. Cad Saúde Pub 2003;19:793-8.

21.Gordilho A, Nascimento JS, Ramos LR, Freire MPA, Espindola $N$, Maia $R$, et al. Desafios a serem enfrentados no terceiro milênio pelo setor saúde na atenção integral ao idoso.Bah An Dados 2001;10:138-53.

22.Born T, Boechat NS. A qualidade dos cuidados ao idoso Institucionalizado. In: Freitas E, Py L, Cançado F, Doll J, Gorzoni M (orgs.). Tratado de Geriatria e Gerontologia. Rio de Janeiro: Guanabara Koogan, 2006, p798-807.

23.Davim RMB, Torres GV, Dantas SMM, Limas ML. Estudo com idosos de instituições asilares no município de Natal/RN: características socioeconômicas e de saúde. Rev Lat Am Enferm 2004;12:518-24.

24. Muchale SM. Cognição e equilíbrio postural na doença de Alzheimer (Tese). São Paulo: FMUSP, 2007, 88p.

25.Webber A. Avaliação da propensão a quedas em idosos institucionalizados e não institucionalizados correlacionando com nível de cognição e equilíbrio (monografia). Cascavel: UNIOESTE, 2004, 47p.

26.Xavier FMF, Ferraz MPT, Bertollucci P, Poyares D, Moriguchi EH. Episódio depressivo maior prevalência e impacto sobre qualidade de vida, sono e cognição em octogenários. Rev Bras Psiquiatr 2001;23:62-70.

27.Schlindwein-Zanini R. Aspectos Psicológicos e neuropsicológicos do idoso. In: Rosa Neto F (Org.). Manual de atividade motora para terceira idade. Porto Alegre: Artmed, 2009, p 6273.

28.Myamoto ST, Lombardi I, Berg KO, Ramos LR, Natour J. Brasilian Vision of the Berg Balance Scale. Braz J Med Biolog Res 2004;37:1411-21.

29.Borges CF, Ferratin AC, Morelli JGS, Rebelatto JR. Qualidade na execução de AVD's em idosos institucionalizados que permaneciam sem sair de suas residências por mais de 6 meses. Rev Bras Fisioter 2005;6:1-9.

30.Canonici AP, Tanaka K, Ferreira F, Stella F, Gobbi S. Análise Comparativa da Capacidade Funcional de Idosos Esquizofrênicos Institucionalizados. Rev Movim 2008;1:11-5. 